\title{
Structure and mixing of a transverse jet in incompressible flow
}

\author{
By J. E. BROADWELL AND R. E. BREIDENTHAL † \\ Graduate Aeronautical Laboratories, California Institute of Technology, Pasadena, CA 91125
}

(Received 27 December 1983 and in revised form 15 June 1984)

The flow field induced by a jet in incompressible cross-flow is analysed and the results compared with those obtained in a reacting water-jet experiment. It is argued that the axial vortex pair in the flow arises from the jet momentum normal to the free stream, the momentum flux being equivalent to a normal force, i.e. to a lift.

\section{Introduction}

The structure, trajectory and mixing rate of transverse jets have been investigated in numerous experiments. The paper of Kamotani \& Greber (1972), for example, contains a useful bibliography and presents additional data describing a striking feature of these flows, namely that the jet quickly takes the form of two counterrotating vortices aligned with the free stream. Most experiments also show that, in the far field, the jet penetration $y$ (the jet centreline distance from the nozzle exit plane) and the vortex spacing $2 R$ vary with the downstream distance $x$ raised to a power close to one-third.

Most theoretical attempts to explain the jet motion involve integral methods, and, of necessity, many simplifying assumptions, particularly with regard to entrainment. Adler \& Baron (1979) discuss much of this work and propose extensions of the method. A difficulty associated with the integral approach is in specifying surface forces, including 'drag', on the control volume containing the jet. This difficulty is circumvented if the flow is treated directly as that of a pair of counter-rotating vortices, an appropriate approximation in the far field. Durando (1971), for example, develops such a semi-empirical model for this region of the flow.

In the analyses referred to above, the presence of the vortex pair is taken to be known from experiment. In the present work, a new view of the flow is presented that explains the origin of the vortices, leads to the experimentally observed coordinate scaling variables, and results in a simple description of the far-field behaviour.

\section{Analysis}

Consider the flow sketched in figure 1 of a jet with density $\rho_{\mathrm{j}}$ and velocity $V_{\mathrm{j}}$ discharging perpendicularly into a stream of density $\rho_{\infty}$ and velocity $V_{\infty}$, both fluids being incompressible. We examine the limiting situation in which the jet momentum flux $m_{\mathrm{j}} V_{\mathrm{j}}$ is held constant as the mass flow $m_{\mathrm{j}}$ and jet diameter $d_{0}$ are reduced to zero while $V_{j}$ increases correspondingly. The limit approached is that of a point source of normal momentum, a 'lift' force of vanishing drag. That a lift generates a pair of

$\dagger$ Present address: Department of Aeronautics and Astronauties, University of Washington, Seattle, WA 98195. 




Figure 1. Flow geometry.

counter-rotating vortices is, of course, well known, the flow about a wing being the most familiar example.

The experiments mentioned above, in which the one-third power law was observed, dealt with flows at high Reynolds number. If viscosity plays no global role and only serves to dissipate energy at the Kolmogorov microscale, then the only global length in the above described limiting flow is

$$
l=\left(\frac{m_{\mathrm{j}} V_{\mathrm{j}}}{\rho_{\infty} V_{\infty}^{2}}\right)^{\frac{1}{2}}
$$

The utility of this parameter in correlating trajectories of actual jets will be illustrated after the far-field behaviour of the vortex pair is derived.

In the far field the vortices move with the free stream and are approximately aligned with it. Therefore, in a coordinate system moving with the free-stream velocity $V_{\infty}$, the flow is approximately two-dimensional in the mean and is that associated with a pair of vortices.

The vertical velocity $\mathrm{d} y / \mathrm{d} t$, the impulse per unit length $P$, the circulation of one vortex $\Gamma$ and the vortex-core separation $2 R$ are related by the equations

$$
\frac{\mathrm{d} y}{\mathrm{~d} t}=\frac{c_{1} \Gamma}{R}, \quad P=c_{2} \rho_{\infty} \Gamma R .
$$

For line vortices $c_{1}=\frac{1}{4} \pi$ and $c_{2}=2$ (see Lamb 1945). Combining these equations, we get

$$
\rho_{\infty} R^{2} \frac{\mathrm{d} y}{\mathrm{~d} t}=c_{3} P
$$

where $c_{3}=c_{1} / c_{2}$. (In the following, the symbol $c$ with various subscripts denotes constants.)

In the far field, i.e. for $x \gg l$, we expect the flow to be independent of $l$ and therefore to find a solution with similarity in which $R$ is proportional to $y$. With this assumption, (4) becomes

$$
c_{4} \rho_{\infty} y^{2} \frac{\mathrm{d} y}{\mathrm{~d} t}=P
$$

This is the equation describing the flow generated by a line impulse of strength $P$ per unit length, and shows that

$$
y=c_{5}\left(\frac{P}{\rho_{\infty}}\right)^{\frac{1}{3}} t^{\frac{1}{3}}
$$


Equations (2) and (3) then yield

$$
R=c_{6}\left(\frac{P}{\rho_{\infty}}\right)^{\frac{1}{3}} t^{\frac{1}{3}}, \quad \Gamma=c_{7}\left(\frac{P}{\rho_{\infty}}\right)^{\frac{2}{3}} t^{-\frac{1}{3}},
$$

Returning to the original coordinate system by use of the transformation $x=V_{\infty} t$, and noting that $P=m_{\mathrm{j}} V_{\mathrm{j}} / V_{\infty}$, we find

$$
\frac{y}{l}=c_{5}\left(\frac{x}{l}\right)^{\frac{1}{3}}
$$

and, from (7) and (8),

$$
\frac{R}{l}=c_{6}\left(\frac{x}{l}\right)^{\frac{1}{3}}, \quad \frac{\Gamma}{V_{\infty} l}=c_{7}\left(\frac{x}{l}\right)^{-\frac{1}{3}} .
$$

These results may be expected to describe the flow fields generated by real jets of high velocity ratio when $x \gg l$. To make a comparison for a case in which $\rho_{j}=\rho_{\infty}$ we note that

$$
l=\left(\frac{m_{\mathrm{j}} V_{\mathrm{j}}}{\rho_{\infty} V_{\infty}^{2}}\right)^{\frac{1}{2}}=\left(\frac{1}{4} \pi\right)^{\frac{1}{2}} r d_{\mathbf{0}},
$$

where $r=V_{\mathrm{j}} / V_{\infty}$, and $V_{\mathrm{j}}$ is taken to be uniform across the nozzle exit. Equation (9) can then be written

$$
\frac{y}{r d_{0}}=c_{8}\left(\frac{x}{r d_{0}}\right)^{\frac{1}{3}}
$$

That the far-downstream penetration varies with $x^{\frac{1}{3}}$ and that lengths scale with $r d_{0}$ has been noted in most experimental investigations. Pratte \& Baines (1967), for example, find that the expression

$$
\frac{y}{r d_{0}}=2.05\left(\frac{x}{r d_{0}}\right)^{0.28}
$$

describes the jet centreline location for $r$ ranging between 5 and $\mathbf{3 5}$ and for two different nozzle diameters.

\section{Mixing and flame length}

If the jet contains a substance that reacts rapidly with one carried in the free stream, then the rate of disappearance of the jet reactant or the appearance of the reaction product is a measure of the rate at which the two streams mix molecularly. When fuel jets burn in air, the length of the flame is the distance required to entrain and mix (again to the molecular level) a stoicheoimetric amount of air. Weddell, in a study of turbulent fuel jets described in Hottel (1953), discharged water jets containing an alkali and a $\mathrm{pH}$ indicator, phenolphthalein, into a stationary acidic reservoir. The jet, which is initially red, becomes colourless when it entrains and mixes with sufficient acid. For these experiments, the normality or equivalence ratio $\phi$, defined as the mass of reservoir fluid required to cause the colour to vanish from a unit mass of jet fluid, was varied by changing the strengths of the two solutions. The flame length in this situation is the distance to colour disappearance. The same technique was applied here to obtain information on mixing rates of jets in cross-flows.

When the equivalence ratio is high so that the flames extend into the far field, the analysis in $\$ 2$ can be used to predict the dependence of length on the various parameters. 
In proposed models of mixing and reaction in turbulent jets in stationary reservoirs (Broadwell 1982) and in turbulent shear layers (Broadwell \& Breidenthal 1982), these processes are taken to begin with large-scale intertwining of the two fluids. This initial step is followed by an inviscid cascade to the Kolmogorov scale $\lambda_{0}$. For the jet in a stationary reservoir, it is argued that the time $t_{\mathrm{d}}$ required for the entrainment of a given quantity of reservoir fluid, its distribution throughout the jet and for the subsequent cascade scales with $d / V$, where $d$ and $V$ are the local jet diameter and velocity. On the other hand, since

$$
\lambda_{0} \sim\left(\frac{\nu^{3} d}{V}\right)^{\frac{1}{4}}
$$

the time $t_{\lambda}$ for diffusion across this distance is expressed by

$$
t_{\lambda} \sim \frac{\lambda_{0}^{2}}{D} \sim\left(\frac{S c}{R e_{a}^{\frac{1}{2}}}\right) \frac{d}{V}
$$

where $D$ is the molecular diffusion coefficient, $S c=\mu / \rho D$ the Schmidt number and $R e_{d}=\rho V d / \mu$. Therefore the ratio $T$ of the diffusion time to the large-scale breakdown time $t_{\mathrm{d}}$ is given by

$$
T=\frac{t_{\lambda}}{t_{\mathrm{d}}} \sim \frac{S c}{R e^{\frac{1}{2}}},
$$

where $R e_{0}$ is the initial Reynolds number equal to $R e_{d}$ (since Reynolds number is constant for a jet in a stationary reservoir). The flame ends when every element of jet fluid is molecularly mixed with at least $\phi$ parts of reservoir fluid. Equation (13) shows that for sufficiently high Reynolds number the diffusion time is fast relative to the entrainment-cascade time, and thus flame length, being controlled by this latter time, is independent of Reynolds number at high values of this parameter. Furthermore, since the entrainment increases linearly with axial distance $x$, the flame length also increases linearly with $\phi$. The water-jet experiments of Weddell, as well as the more detailed recent studies of Dahm, Dimotakis \& Broadwell (1984), yield results in agreement with this analysis.

In applying these ideas to the jet in a cross-flow, we note first that the appropriate far-field Reynolds number is $\Gamma / \nu \sim R e_{l}(l / x)^{\frac{1}{3}}$ from (11), where

$$
R e_{l}=\frac{V_{\infty} l}{\nu}=\left(\frac{\pi}{4} \frac{\rho_{\mathrm{j}}}{\rho_{\infty}}\right)^{\frac{1}{2}} \frac{V_{\mathrm{j}} d_{0}}{\nu}
$$

Thus for the following analysis to hold, the initial Reynolds number must be sufficiently high for the flame to end before the Reynolds number falls to a level at which the flow becomes laminar.

In a transverse flow, with $\rho_{\mathrm{j}}=\rho_{\infty}$, a flame of equivalence ratio $\phi$ ends when the ratio of the initial jet flux to the total flux of molecularly mixed fluid in the jet reaches $(\phi+1)^{-1}$, i.e. when

$$
\frac{V_{\infty} R^{2}}{V_{\mathrm{j}} A_{0}} \sim \phi+1
$$

where the jet area is taken to scale with the vortex spacing $R$ and $A_{0}$ is the initial jet area.

We argue in the Appendix that the microscale is reached at a time after entrainment proportional to the instantaneous vortex rotation period, which in turn is proportional to the Lagrangian vortex age. Thus $R /(\mathrm{d} y / \mathrm{d} t) \sim t$, with the corresponding cascade distance $\sim V_{\infty} t$. That is, the cascade distance beginning at a given axial station is proportional to the axial location of that station itself. Hence the 
cascade distance is some multiple of the distance for the entrainment of a given amount of reservoir fluid.

Combining (10) and (14) and using the definition of $l$, we get for the flame length

$$
x_{\mathrm{f}}=c_{9}\left(\frac{V_{\infty}}{V_{\mathrm{j}}}\right)^{\frac{1}{2}}(\phi+1)^{\frac{3}{2}} A_{0}^{\frac{1}{2}}
$$

independent of the Schmidt and Reynolds numbers.

Since the conditions have been taken to be such that the flame ends in the far field, no distinction was made between the chord length $\left(x_{\mathrm{f}}^{2}+y_{\mathrm{f}}^{2}\right)^{\frac{1}{2}}$ and the axial length $x_{\mathrm{f}}$ of the flame.

\section{Experiment}

An aqueous jet was introduced through the test-section ceiling of a water tunnel, which provided the cross-flow (figure 1). The water-tunnel apparatus is described elsewhere (Breidenthal 1981). Briefly, the test section is $7 \mathrm{~cm}$ high $\times 11 \mathrm{~cm}$ span $\times 45 \mathrm{~cm}$ long, through which a transparent solution of sulphuric acid flowed. A jet nozzle of $0.20 \mathrm{~cm}$ diameter injected a red solution of sodium hydroxide and phenolphthalein, a $\mathrm{pH}$ indicator. When the two fluids mixed, a rapid chemical reaction caused the red, injected solution to disappear. The volume equivalence ratio $\phi$ (the volume ratio of ambient to injected fluid required to effect the transformation) was varied by changing the concentrations of the acid and base. The momentum flux of the jet was estimated from the measured volume flow rate. No correction was made for the nozzle boundary layers. The jet Reynolds numbers $R e_{0}$ varied between $10^{2}$ and $10^{4}$. The coordinates at the end of the red 'flame' were estimated visually. 'This measurement was of course somewhat subjective, but the flame end was reasonably well defined and the measurements were made in a consistent fashion.

Figure 2 shows the flame chord length $C=\left(x_{\mathrm{f}}^{2}+y_{\mathrm{f}}^{2}\right)^{\frac{1}{2}}$ as a function of $V_{\infty} / V_{\mathrm{j}}$ for several values of $\phi$. The flame length at $V_{\infty}=0$ is taken from Weddell for all values of $\phi$ except $\phi=1.1$, since the water channel was too narrow to accommodate the jet at low values of $V_{\infty} / V_{\mathrm{j}}$. The flame chord length exhibits a minimum at or below $V_{\infty} / V_{\mathrm{j}}=0.06$, indicating very rapid mixing there.

The downstream flame length $x_{\mathrm{f}}$ is shown in figure 3 , where $x_{\mathrm{f}} / d_{0} \sim\left(V_{\infty} / V_{\mathrm{j}}\right)^{\frac{1}{2}}$. In figure 4 the measurements are compared with the analysis in $\S 3$. There is considerable scatter, but the data generally straddle a horizontal line as predicted by the theory, at least for $V_{\infty} / V_{\mathrm{j}} \geqslant 0.1$. The data for $\phi=1.1$ do not follow the pattern, presumably because the flame lengths are too short for far-field behaviour, and these data points are not shown in figure 4 .

\section{Discussion}

The flame length is independent of the Reynolds number $R e_{0}$ (for fixed $V_{\infty} / V_{\mathrm{j}}$ and $\phi$ ) above a certain critical value. All data shown are from this regime. For a lower Reynolds number the flame length was much longer. This behaviour is typical of mixing in turbulent flows, and is due, it seems clear, to the sensitivity of the mixing rate to the presence of small-scale turbulent motions. As long as the small scales are present, they generate sufficient reaction interface area to mix rapidly all reactant that is broken down to their scale. Thus the breakdown process (which is inviseid) limits the mixing rate. But, when the small scales are not sufficiently developed at lower $R e$, the diffusion process at the reaction interface determines the mixing rate. 


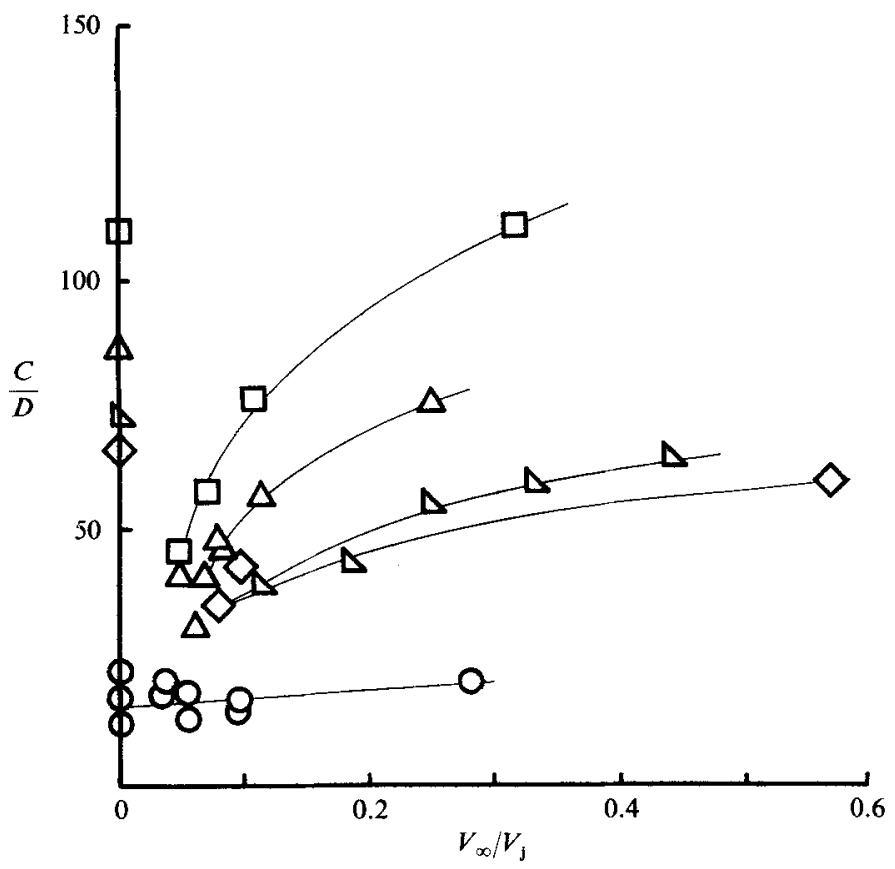

Figure 2. Chord length of the 'flame' for various values of equivalence ratio $\phi=11(\square) ; 8.7(\triangle) ; 7.3(\Delta) ; 6.7(\diamond) ; 1.1(\bigcirc)$.

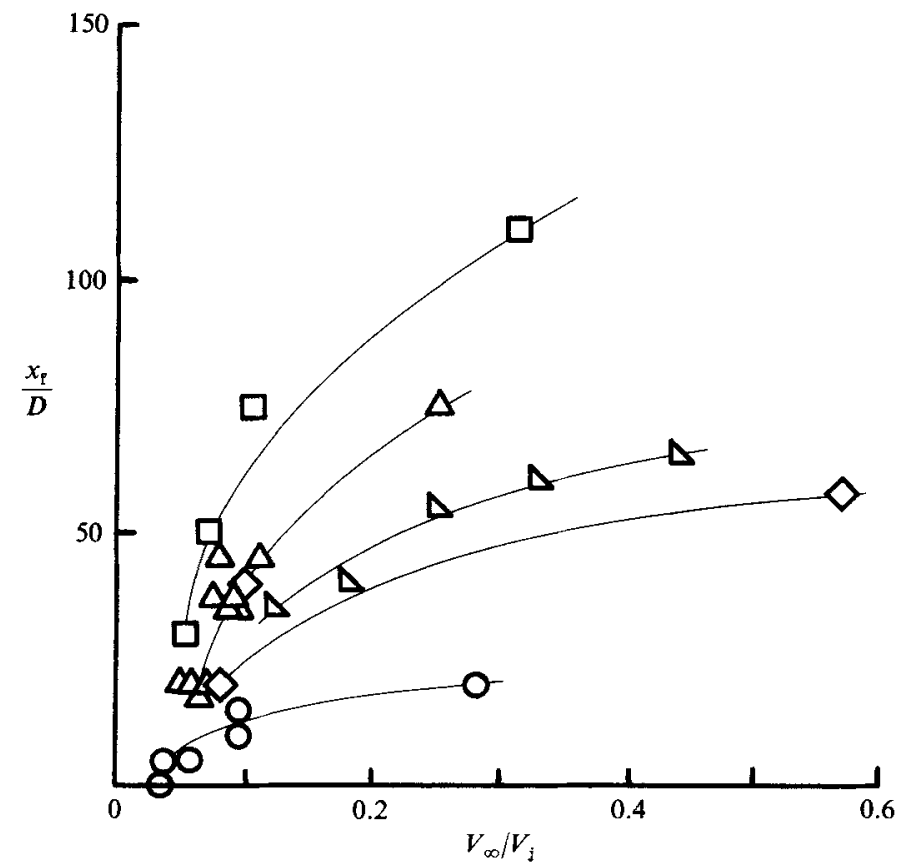

Figure 3. Downstream flame length. Notation as in figure 2. 


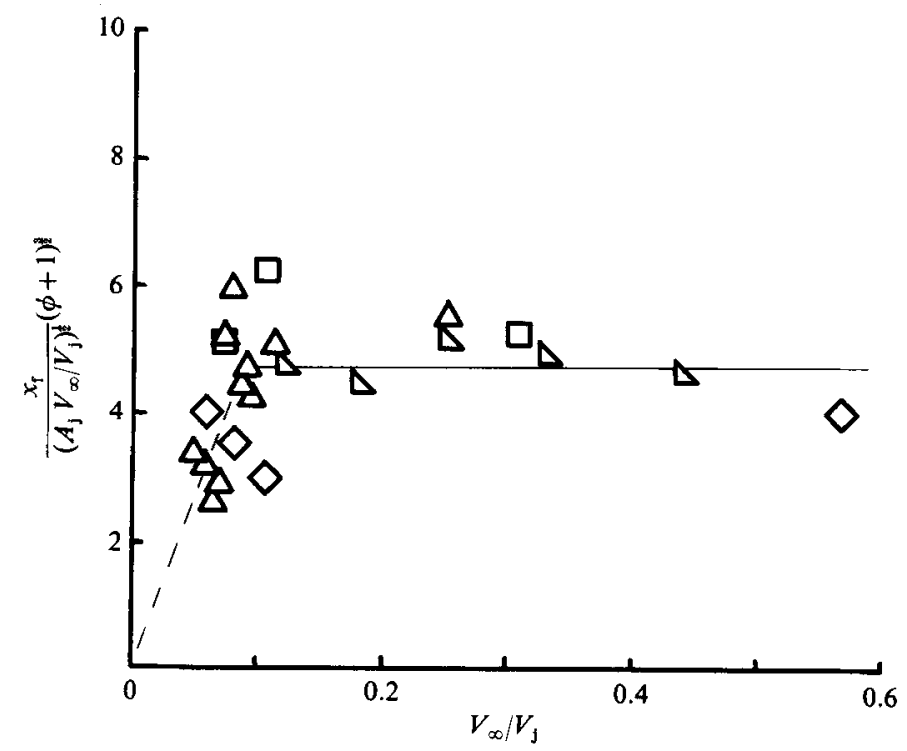

Figure 4. Normalized downstream flame length. Notation as in figure 2.

Because Re determines interface properties such as area and strain rate, the mixing is a strong function of $R e$ below the critical value. A rough estimate of this critical Reynolds number at the flame tip is $\Gamma_{\mathrm{p}} / \nu=3 \times 10^{2}$.

The behaviour of the flame chord length for small $V_{\infty} / V_{j}$ may have been expected in that the (far-field) model predicts that the flame length goes to zero as $V_{\infty} / V_{j}$ vanishes. Of course, the flame length is finite at $V_{\infty} / V_{j}=0$, and a transition is required. There are two questions about this transition. First, it is surprising that the observed behaviour seems to match the far-field model even though the flame length is not long compared with the characteristic length $l$ at the transition. Secondly, the precise physical reason for the transition is unknown. Somehow the structure of the axisymmetric jet is altered by the cross-flow, and the resulting vorticity field is a more efficient mixer.

The explanation for the structural transition as $V_{\infty}$ is increased from zero may be associated with the formation of the counter-rotating vortex pair or with a TaylorGörtler instability of the curved upstream side of the jet. It was observed that the resulting vortices were relatively large, consistent with the large growth rate of a curved shear layer (Bradshaw 1969). Such an enhanced vortex growth rate over the axisymmetric case might imply more rapid mixing and a shorter flame length.

\section{Concluding remarks}

The analysis and the comparison of the results with observation have concentrated on the far-field behaviour of transverse jets. It is unfortunate that a near-field description of the flow generated by a point force is not available (to the authors' knowledge). It would be interesting to compare the early evolution of the vortices in this flow with those produced by jets, in which ease vorticity arises from the interaction of the cross-flow with the jet.

The measured flame length of the transverse jets is in reasonble agreement with a simple description of the far-field behaviour. The most notable observation is a 
minimum in flame chord length at a velocity ratio of about $V_{\infty} / V_{j}=0.06$. While the existence of such a minimum is anticipated from the analysis, the corresponding transformation in the flow structure remains unclear.

The authors are pleased to acknowledge support for this work by the United States Office of Naval Research under contract N0014-76-C-0260.

\section{Appendix}

In all self-similar flows the rotation rate of the global vortical motions varies as $t^{-1}$. Dimensional considerations require this for all flows with no imposed lengthscale. That is to say, there is only one time on which all global properties must depend, the elapsed time. As an example, consider a constant-density free turbulent jet into a quiescent fluid. The lengthscale $\delta$ is proportional to $x$, and conservation of momentum implies

$$
U \sim x^{-1} .
$$

To get the Lagrangian dependence of vorticity on time, we write

$$
U=\frac{\mathrm{d} x}{\mathrm{~d} t} \sim x^{-1} \quad \text { or } \quad x \sim t^{\frac{1}{2}}
$$

Therefore

$$
\frac{\mathrm{d} x}{\mathrm{~d} t} \sim t^{-\frac{1}{2}}
$$

and the global rotation rate

$$
\Omega \sim \frac{\mathrm{d} x}{\mathrm{~d} t} / \delta \sim t^{-1}
$$

A reference table of velocity and length dependence on $x$ for other classical flows can be found in Tennekes \& Lumley (1972) and Cantwell (1981).

\section{REFERENCES}

Adler, D. \& Baron, A. 1979 Prediction of a three-dimensional circular turbulent jet in cross flow. $A I A A J .17,168$.

Bradshaw, P. 1969 The analogy between streamline curvature and buoyancy in turbulent shear flow. J. Fluid Mech. 36, 177.

Breidenthat, R. 1981 structure in turbulent mixing layers and wakes using a chemical reaction. J. Fluid Mech. 109, 1.

Broadwell, J. E. 1982 A model of turbulent diffusion flames and nitric oxide generation, Part I. TRW Rep. 38515-6001-U'T-00.

Broadwell, J. E. \& Breidenthal, R. E. 1982 A simple model of mixing and chemical reaction in a turbulent shear layer. J. Fluid Mech. 125, 397.

Cantwell, B. J. 1981 Organized motion in turbulent flow. Ann. Rev. Fluid Mech. 13, 457.

Dahm, W. J., Dimotakis, P. E. \& Broadwell, J. E. 1984 Non-premixed turbulent jet flames. AIAA Paper 84-0369.

Durando, N. A. 1971 Vortices induced in a jet by a subsonic cross flow. $A I A A J .9,325$.

Hotтel, H. C. 1953 Burning in laminar and turbulent fuel jets. In Proc. 4th Symp. (Intl) on Combustion, p. 97. Williams \& Williams.

Kamotani, Y. \& Greber, I. 1972 Experiments on a turbulent jet in a cross flow. $A I A A J .10$, 1425.

LAMB, H. 1945 Hydrodynamics, 6th edn. Dover.

Pratte, B. D. \& Baines, W. D. 1967 Profiles of the round turbulent jet in a cross flow. $J$. Hydronaut. Div. ASCE 92, 53.

Tennekes, H. \& Lumley, J. E. 1972 A First Course in Turbulence. MIT Press. 